\title{
Effective Onsite Interaction for Dynamical Mean-Field Theory
}

\author{
Yusuke Nomura ${ }^{1}$, Merzuk Kaltak ${ }^{2}$, Kazuma Nakamura ${ }^{1,3}$, Ciro Taranto ${ }^{4}$, Shiro Sakai ${ }^{5}$, \\ Alessandro Toschi ${ }^{4}$, Ryotaro Arita ${ }^{1,3,6}$, Karsten Held ${ }^{4}$, Georg Kresse ${ }^{2}$, and Masatoshi Imada ${ }^{1,3}$ \\ ${ }^{1}$ Department of Applied Physics, University of Tokyo, \\ 7-3-1 Hongo, Bunkyo-ku, Tokyo, 113-8656, Japan \\ ${ }^{2}$ University of Vienna, Faculty of Physics and Center for Computational \\ Materials Science, Sensengasse 8/12, A-1090 Vienna, Austria \\ ${ }^{3}$ JST CREST, 7-3-1 Hongo, Bunkyo-ku, Tokyo, 113-8656, Japan \\ ${ }^{4}$ Institute for Solid State Physics, Vienna University of Technology, A-1040 Vienna, Austria \\ ${ }^{5}$ Centre de Physique Théorique, École Polytechnique, CNRS, 91128 Palaiseau Cedex, France and \\ ${ }^{6}$ JST-PRESTO, Kawaguchi, Saitama, 332-0012, Japan
}

(Dated: November 9, 2018)

\begin{abstract}
A scheme to incorporate non-local polarizations into the dynamical mean-field theory (DMFT) and a tailor-made way to determine the effective interaction for the DMFT are systematically investigated. Applying it to the two-dimensional Hubbard model, we find that non-local polarizations induce a non-trivial filling-dependent anti-screening effect for the effective interaction. The present scheme combined with density functional theory offers an $a b$ initio way to derive effective onsite interactions for the impurity problem in DMFT. We apply it to $\mathrm{SrVO}_{3}$ and find that the anti-screening competes with the screening caused by the off-site interaction.
\end{abstract}

PACS numbers: 71.10.-w, 71.27.+a

-Introduction. Understanding physical properties of strongly correlated electron systems is one of the most challenging subjects in condensed matter physics [1, 2]. For this purpose, it is essential to capture fermionic many-body effects necessitating a proper and accurate treatment of a large number of interacting fermions. The large number of electronic degrees of freedom in real materials are intractable, even with rapidly developing computational power. Hence, various ingenious ways of reducing the degrees of freedom have been developed. Aside from the reduction to mean-field effective oneparticle Hamiltonians, as in density functional theory (DFT), including dynamical fluctuations for the reduced and tractable degrees of freedom is a route that has been explored extensively over the last decades.

Approaches have been proposed [1 $[3]$ to partially trace out the degrees of freedom far from the Fermi level, leaving an effective low-energy model for a small number of bands near the Fermi level. The resulting Hubbard-type lattice fermion models are much simpler than the original problem containing a huge number of bands. This reduction (downfolding) has been successfully incorporated in the constrained random phase approximation (cRPA) 4. by the use of maximally localized Wannier orbitals (MLWO) [5] as a basis set. It should be noted that, by tracing out certain electronic degrees of freedom, the effective interactions in the lattice fermion models (e.g. the Hubbard $U$, as exemplified by $U^{\text {cRPA }}$ derived with the cRPA) are much reduced compared to the original bare Coulomb interactions [6 14 because of the screening by polarizations of the eliminated degrees of freedom.

Although several efficient ways to solve the lattice fermion models have been proposed [1], it is still too difficult to treat realistic situations so that a further re- duction is highly desired. The widely used dynamical mean-field theory (DMFT) [15, 16] indeed offers a practical way of describing local correlation effects along this line [2], where the lattice fermion models are mapped onto quantum impurity models.

Although $U^{\text {cRPA }}$ is widely used as input for DMFT calculations, the conventional cRPA treatment totally excludes non-local screening processes within the target band. These are also not contained in the DMFT, which only accounts for the local screening processes. Hence, in the present work we argue that a better starting point is the inclusion of non-local screening processes of the target band within the RPA yielding an effective onsite interaction $U^{\mathrm{DMFT}}$. Albeit tailor-made interaction parameters for the impurity problem were employed in Ref. 17, a systematic investigation has been missing so far.

In this Letter, we examine a scheme for the systematic determination of the effective onsite interaction $U^{\mathrm{DMFT}}$ for DMFT calculations. This scheme is applied to both the two-dimensional (2D) single-band Hubbard model and to $\mathrm{SrVO}_{3}$ by using an ab initio description. The application to the Hubbard model unexpectedly reveals the inequality $U^{\mathrm{DMFT}}>U$ and a non-trivial filling dependence of $U^{\mathrm{DMFT}}$ with a peak around the van Hove singularity. A filling-dependent $U^{\mathrm{DMFT}}$ is also observed in the ab initio results for $\mathrm{SrVO}_{3}$. These are ascribed to an anti-screening effect induced by non-local polarizations, namely, a test-charge electron induces an off-site hole or electron and they again induce an onsite electron. This nonlocal effect increases $U^{\mathrm{DMFT}}$. The present elucidation contributes not only to the specific determination of the DMFT-interaction parameters, but also to gain insight into the nature of the reduced and simplified fermionic models in general. 
-Equations to derive $U^{\mathrm{DMFT}}$. Here, we derive the basic equations to evaluate $U^{\mathrm{DMFT}}$ from first principles calculations 17]. In the RPA, the screened Coulomb interaction $W$ can be written as $\left(1-v \chi_{0}\right)^{-1} v$ with the independentparticle polarization $\chi_{0}$ and the bare Coulomb interaction $v$. The polarization $\chi_{0}$ is divided into $\chi_{0}^{t}$ and $\chi_{0}^{r}$, where $\chi_{0}^{t}$ is a polarization formed in the target subspace and $\chi_{0}^{r}$ is the rest. Note that this decomposition is not necessarily restricted to bands (cRPA); it is also applicable to the real space using localized basis sets. For example, the "dimensional downfolding" has been formulated to derive effective models in reduced dimensions such as $2 \mathrm{D}$ or $1 \mathrm{D}$ models by excluding polarizations within the target layer/chain [8]. With this decomposition and within the RPA, the fully screened $W$ can be obtained in a two-step procedure as [4]

$$
\bar{W}=\left(1-v \chi_{0}^{r}\right)^{-1} v
$$

and

$$
W=\left(1-\bar{W} \chi_{0}^{t}\right)^{-1} \bar{W},
$$

where $\bar{W}$ describes a screened Coulomb interaction excluding a specified subset of excitations $\chi_{0}^{t}$. These excitations are taken into account when the effective model with the interaction $\bar{W}$ is solved. Alternatively, $\bar{W}$ is obtained from the fully screened $W$, by rewriting Eq. (2) [17] as

$$
\bar{W}=W\left(1+\chi_{0}^{t} W\right)^{-1} .
$$

In the present scheme, $\bar{W}$ corresponds to $U^{\mathrm{DMFT}}$ and $\chi_{0}^{t}$ is a one-center or local target polarization formed at the impurity site.

In practice, the static independent-particle polarization formed in the target bands $(t b)$ is calculated using

$$
\chi_{0}^{t b}\left(\mathbf{r}, \mathbf{r}^{\prime}\right)=2 \sum_{\alpha \beta}^{\epsilon t b} \sum_{\mathbf{q} \mathbf{k}} \frac{f_{\beta \mathbf{k}+\mathbf{q}}-f_{\alpha \mathbf{k}}}{\epsilon_{\beta \mathbf{k}+\mathbf{q}}-\epsilon_{\alpha \mathbf{k}}} \psi_{\alpha \mathbf{k}}^{*}(\mathbf{r}) \psi_{\beta \mathbf{k}+\mathbf{q}}(\mathbf{r}) \psi_{\beta \mathbf{k}+\mathbf{q}}^{*}\left(\mathbf{r}^{\prime}\right) \psi_{\alpha \mathbf{k}}\left(\mathbf{r}^{\prime}\right),
$$

where $\left\{\psi_{\alpha \mathbf{k}}, \epsilon_{\alpha \mathbf{k}}\right\}$ are one-body wavefunctions and their energies with the wave vector $\mathbf{k}$ and the band index $\alpha$. The factor of 2 comes from the spin sum. The band summation is performed only over the target bands in the effective model. Since the Bloch wavefunctions are related to the Wannier functions via the unitary transform as

$$
\psi_{\mathrm{ok}}(\mathbf{r})=\frac{1}{\sqrt{N}} \sum_{m i \mathbf{R}} e^{i \mathbf{k} \cdot \mathbf{R}} U_{m i, \alpha}^{\dagger(\mathbf{k})} \phi_{m i \mathbf{R}}(\mathbf{r}),
$$

the polarization can be recast as

$$
\begin{aligned}
\chi_{0}^{t b}\left(\mathbf{r}, \mathbf{r}^{\prime}\right)= & \frac{2}{N^{2}} \sum_{m n o p} \sum_{i j k l} \sum_{\mathbf{R}_{1}-\mathbf{R}_{4}}\left[\sum_{\alpha \beta}^{\epsilon t b} \sum_{\mathbf{q k}} \frac{f_{\beta \mathbf{k}+\mathbf{q}}-f_{\alpha \mathbf{k}}}{\epsilon_{\beta \mathbf{k}+\mathbf{q}}-\epsilon_{\alpha \mathbf{k}}} e^{-i \mathbf{k} \cdot\left(\mathbf{R}_{1}-\mathbf{R}_{4}\right)}\right. \\
& \left.\times e^{i(\mathbf{k}+\mathbf{q}) \cdot\left(\mathbf{R}_{2}-\mathbf{R}_{3}\right)}\left(U_{m i, \alpha}^{\dagger(\mathbf{k})}\right)^{*} U_{n j, \beta}^{\dagger(\mathbf{k}+\mathbf{q})}\left(U_{o k, \beta}^{\dagger(\mathbf{k}+\mathbf{q})}\right)^{*} U_{p l, \alpha}^{\dagger(\mathbf{k})}\right] \\
& \times \phi_{m i \mathbf{R}_{1}}^{*}(\mathbf{r}) \phi_{n j \mathbf{R}_{2}}(\mathbf{r}) \phi_{o k \mathbf{R}_{3}}^{*}\left(\mathbf{r}^{\prime}\right) \phi_{p l \mathbf{R}_{4}}\left(\mathbf{r}^{\prime}\right),
\end{aligned}
$$

where $m-p, i-l, \mathbf{R}_{1}-\mathbf{R}_{4}$ are the orbital, primitive site, superlattice site indices respectively and $N$ indicates the total number of superlattice sites. With this expression, we specify the target-band polarization formed at the impurity site (the 0th site in $\mathbf{R}=\mathbf{0}$ ) as

$$
\chi_{0}^{\mathrm{imp}}\left(\mathbf{r}, \mathbf{r}^{\prime}\right)=\sum_{m n o p} C_{m m o p} \phi_{m 0 \mathbf{0}}^{*}(\mathbf{r}) \phi_{n 00}(\mathbf{r}) \phi_{o 00}^{*}\left(\mathbf{r}^{\prime}\right) \phi_{p 00}\left(\mathbf{r}^{\prime}\right)
$$

with

$C_{m \text { mop }}=\frac{2}{N^{2}} \sum_{\alpha \beta}^{\epsilon t b} \sum_{\mathbf{q k}} \frac{f_{\beta \mathbf{k}+\mathbf{q}}-f_{\text {ck }}}{\epsilon_{\beta \mathbf{k}+\mathbf{q}}-\epsilon_{\alpha \mathbf{k}}}\left(U_{m 0, \alpha}^{\dagger(\mathbf{k})}\right)^{*} U_{n 0, \beta}^{\dagger(\mathbf{k}+\mathbf{q})}\left(U_{o 0, \beta}^{\dagger(\mathbf{k}+\mathbf{q})}\right)^{*} U_{p 0, \alpha}^{\dagger(\mathbf{k})}$

corresponding to the local one-center components of a polarization matrix in the Wannier orbital basis. Now, by identifying $\chi_{0}^{t}$ in Eq. (3) as $\chi_{0}^{\mathrm{imp}}$ and $\bar{W}$ as $U^{\mathrm{DMFT}}$, we write the Dyson equation for the effective interaction as

$$
\begin{gathered}
W\left(\mathbf{r}, \mathbf{r}^{\prime}\right)=U^{\mathrm{DMFT}}\left(\mathbf{r}, \mathbf{r}^{\prime}\right)+\int \mathrm{d} \mathbf{r}^{\prime \prime} \int \mathrm{d} \mathbf{r}^{\prime \prime \prime} U^{\mathrm{DMFT}}\left(\mathbf{r}, \mathbf{r}^{\prime \prime}\right) \chi_{0}^{\mathrm{imp}}\left(\mathbf{r}^{\prime \prime}, \mathbf{r}^{\prime \prime \prime}\right) \\
\times W\left(\mathbf{r}^{\prime \prime \prime}, \mathbf{r}^{\prime}\right) .
\end{gathered}
$$

Multiplying this equation by $\phi_{m 00}^{*}(\mathbf{r}) \phi_{n 00}(\mathbf{r}) \phi_{o 00}^{*}\left(\mathbf{r}^{\prime}\right)$ $\times \phi_{p 00}\left(\mathbf{r}^{\prime}\right)$ and integrating over $\mathbf{r}$ and $\mathbf{r}^{\prime}$, we have

$$
W_{\mu \nu}=U_{\mu \nu}^{\mathrm{DMFT}}+\sum_{\mu^{\prime} \nu^{\prime}} U_{\mu \mu^{\prime}}^{\mathrm{DMFT}} C_{\mu^{\prime} \nu^{\prime}} W_{\nu^{\prime} \nu}
$$

where we introduce a composite index $(\mu, \nu)=\{(m n),(o p)\}$ and the matrix element of $\mathcal{O}=\left\{W, U^{\mathrm{DMFT}}\right\}$ is given by

$\mathcal{O}_{\text {mmop }}=\int \mathrm{d} \mathbf{r} \int \mathrm{d} \mathbf{r}^{\prime} \phi_{m 00}^{*}(\mathbf{r}) \phi_{n 00}(\mathbf{r}) \mathcal{O}\left(\mathbf{r}, \mathbf{r}^{\prime}\right) \phi_{o 00}^{*}\left(\mathbf{r}^{\prime}\right) \phi_{p 00}\left(\mathbf{r}^{\prime}\right)$.

Thus, Eq. (10) is rewritten in a matrix form as

$$
\mathbf{U}^{\mathrm{DMFT}}=\mathbf{W}(\mathbf{1}+\mathbf{C W})^{-1} .
$$

The equation resembles the unscreening equation (3), but it is formulated entirely in terms of "local" one-center quantities, that can be evaluated straightforwardly, allowing for a computationally efficient treatment.

-Application to the Hubbard model. We first apply this scheme to the derivation of $U^{\mathrm{DMFT}}$ for the 2D single-band Hubbard model. This is helpful to get insight into the behavior of $U^{\mathrm{DMFT}}$ with respect to changes of the electron filling. The Hubbard Hamiltonian reads

$$
\mathcal{H}=-t \sum_{\langle i j\rangle \sigma} c_{i \sigma}^{\dagger} c_{j \sigma}-t^{\prime} \sum_{\langle\langle i j\rangle \sigma} c_{i \sigma}^{\dagger} c_{j \sigma}-\mu \sum_{i \sigma} n_{i \sigma}+U \sum_{i} n_{i \uparrow} n_{i \downarrow}
$$

where $c_{i \sigma}^{\dagger}\left(c_{i \sigma}\right)$ creates (annihilates) an electron with spin $\sigma$ at site $i$ and $n_{i \sigma} \equiv c_{i \sigma}^{\dagger} c_{i \sigma} . t\left(t^{\prime}\right)$ is a transfer integral to the (next-)nearest neighbor sites in the $\langle i, j\rangle(\langle\langle i, j\rangle\rangle)$ sums. $U(=8 t)$ and $\mu$ represent the onsite Coulomb repulsion and chemical potential, respectively. Taking into 
account the contributions from the charge susceptibility only (hence being in accordance with $a b$ initio methods), the unscreening equation corresponding to Eq. (11) becomes [18]

$$
U^{\mathrm{DMFT}}=\frac{U}{2}+\frac{\tilde{W}}{1-A \tilde{W}}
$$

Here $\tilde{W}$ is a diagonal element of a real-space $N \times N$ ma$\operatorname{trix} \tilde{\mathbf{W}}=\left(\mathbf{1}-\tilde{\mathbf{U}} \boldsymbol{\chi}_{0}\right)^{-1} \tilde{\mathbf{U}}$, $\tilde{\mathbf{U}}$ a diagonal matrix with elements $\tilde{U}=U / 2$ and $-A$ (with $A>0$ ) the diagonal element of the real-space polarization matrix $\chi_{0}$ with elements $\left(\chi_{0}\right)_{i j}=\chi_{0}\left(\mathbf{R}_{i}-\mathbf{R}_{j}\right)$. The latter is obtained by the Fourier transform of the reciprocal-space static polarization function

$$
\chi_{0}(\mathbf{q})=\frac{2}{N} \sum_{\mathbf{k}} \frac{f_{\mathbf{k}+\mathbf{q}}-f_{\mathbf{k}}}{\xi_{\mathbf{k}+\mathbf{q}}-\xi_{\mathbf{k}}} .
$$

with $\xi_{\mathbf{k}}=-2 t\left(\cos k_{x}+\cos k_{y}\right)-4 t^{\prime} \cos k_{x} \cos k_{y}-\mu$ and $f_{\mathbf{k}}$ being the eigenvalue and the Fermi distribution function, respectively.

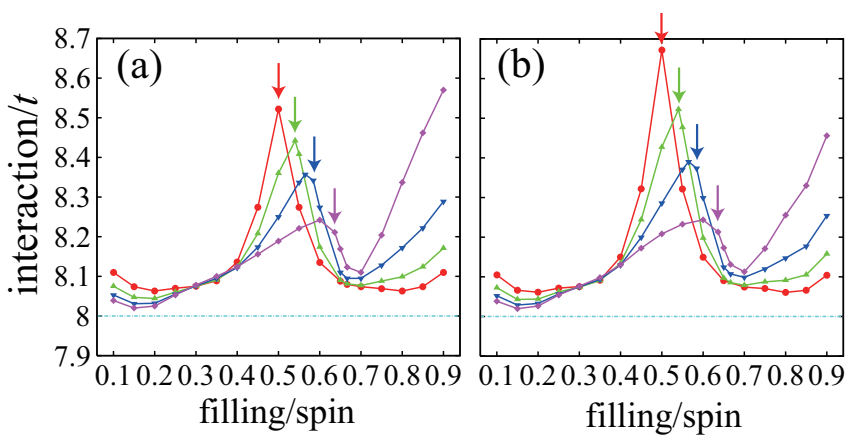

FIG. 1: (color online) Filling dependence of $U^{\mathrm{DMFT}}$ calculated (a) with Eq. (12) and (b) with the approximation [Eq. (14)] for $t^{\prime}=0$ (red), $0.1 t$ (green), $0.2 t$ (blue), and $0.3 t$ (purple). The arrows indicate the fillings at which the van Hove singularity resides at the chemical potential.

Figure 1(a) shows the filling dependence of $U^{\mathrm{DMFT}} / t$ with various $t^{\prime}$. Contrary to a naive expectation, $U^{\mathrm{DMFT}}$ is larger than $U$. Furthermore, the filling dependence of $U^{\mathrm{DMFT}}$ is not monotonic and depends on $t^{\prime}$. For $t^{\prime}=0$, $U^{\mathrm{DMFT}}$ has a strong peak at half filling where the van Hove singularity resides at the Fermi energy. With increasing $t^{\prime}$, the peak shifts to higher filling with reduced peak height, and another rapid increase emerges at further higher filling.

These filling and $t^{\prime}$ dependences of $U^{\mathrm{DMFT}}$ are well understood by the second-order approximation in $\left\{B_{n}\right\}[18]$ :

$$
U^{\mathrm{DMFT}} \sim U+\sum_{n=1}^{N-1}\left(\tilde{U} B_{n}\right)^{2} \frac{\tilde{U}}{1+\tilde{U} A},
$$

where $B_{n} \equiv\left(\chi_{0}\right)_{i, i+n}$ is the non-local contribution to the polarization. Since the second term of the right hand is always positive, the inequality $U^{\mathrm{DMFT}}>U$ holds. Figure 1(b) shows the results of $U^{\mathrm{DMFT}}$ calculated with Eq. (14) for various fillings and $t^{\prime}$. We see in Fig. 1(b) that Eq. (14) well reproduces the overall trend in Fig. 1(a).

The inequality $U^{\mathrm{DMFT}}>U$ reveals anti-screening induced by non-local polarizations $\left\{B_{n}\right\}$. This antiscreening is intuitively understood as follows: Suppose that a test charge electron is put on the impurity site. The local polarization screens this electron by creating holes at the impurity site. On the other hand, the nonlocal polarizations induce holes or electrons at other sites. Then, in the second order process, the induced charges create electrons at the impurity site, enhancing the effective repulsion. Since $\frac{\tilde{U}}{1+\tilde{U} A}$ in Eq. (14) varies smoothly with filling [19], the non-local polarizations $\left\{B_{n}\right\}$ indeed dominate the peculiar filling dependence of $U^{\mathrm{DMFT}}$.

In real materials, off-site Coulomb interactions may play a role. To see this effect, we have studied $U^{\mathrm{DMFT}}$ for a model with the off-site interaction $1 / \epsilon r$ with varying $\epsilon$. We find that the overall filling dependence of $U^{\mathrm{DMFT}}$ is basically the same as that of the Hubbard model while decreasing $\epsilon$ (i.e., increasing off-site interaction) causes an appreciable reduction of $U^{\mathrm{DMFT}}$ (not shown). The longrange Coulomb interactions connect the onsite polarizations at different sites and thus bring about the screening to the impurity-site interaction. Note that this screening works from the zeroth order in $\left\{B_{n}\right\}$; the approximated $U^{\mathrm{DMFT}}$ without the contributions from $\left\{B_{n}\right\}$ indeed becomes smaller than $U$ and has only a weak filling dependence.

-Application to $\mathrm{SrVO}_{3}$. We next present ab initio results of $\mathbf{U}^{\mathrm{DMFT}}$ for $\mathrm{SrVO}_{3}$. This material is a $d^{1}$ metal and one of the most benchmarked systems within LDA+DMFT (local density approximation plus DMFT) [20]. On the basis of the DFT band structure, we define the target bands by the low-energy $t_{2 g}$ bands as was done in Ref. [6]. We construct three MLWOs per $\mathrm{V}$ site from the $t_{2 g}$ Bloch states and calculate $U^{\mathrm{DMFT}}$ for these three orbitals. The implementation details and the convergence checks are elaborated in Ref. [18].

Table \ compares the values of the onsite intra- and inter-orbital Coulomb repulsions $\left(U\right.$ and $\left.U^{\prime}\right)$ and Hund's rule coupling $(J)$ for the bare $(\mathbf{v})$, cRPA $\left(\mathbf{U}^{\mathrm{cRPA}}\right)$ [10], $\mathbf{U}^{\mathrm{DMFT}}$, and full-RPA $(\mathbf{W})$ interactions. The bare Coulomb interactions $(\sim 15 \mathrm{eV})$ are largely screened by the high-energy bands, to give $U^{\mathrm{cRPA}} \sim 3 \mathrm{eV}$. In the present case of $\mathrm{SrVO}_{3}, U^{\mathrm{DMFT}}$ turns out to have a value similar to $U^{\mathrm{cRPA}}$.

The situation changes drastically, however, when we increase the filling $n$ within the rigid-band approximation. The left and right panels in Fig. 2 plot $U$ and $U^{\prime}$, respectively, against the filling $n$. For comparison, we also show the results without the non-local polarizations 
involving the impurity site, i.e., the interaction parameters calculated without the local one-center and "wing" components of the polarization matrix in the Wannier basis ("no-wing" method) 21]. The result is denoted as

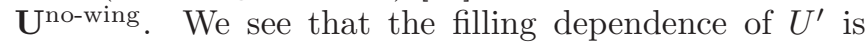
similar to that of $U$, except for a constant shift.

As the filling $n$ increases from $1, U^{\mathrm{DMFT}}$ increases more rapidly than $U^{\mathrm{CRPA}}$. This suggests that the nonlocal anti-screening effect increases more rapidly than the screening. Around $n=2, U^{\mathrm{DMFT}}$ turns to decrease, crossing $U^{\mathrm{cRPA}}$ at $n \sim 3.5$. Finally around the filling end $n \sim 5$, $U^{\mathrm{DMFT}}$ again increases, as seen in the Hubbard model. We see $U^{\text {no-wing }}<U^{\mathrm{DMFT}}$ at all fillings. This is consistent with the model analysis: The non-local contributions to the screening induce an anti-screening and lead to the increase of the onsite interaction. $U^{\text {no-wing }}$ is also smaller than $U^{\text {cRPA }}$ and only weakly depends on the filling, consistently with the model analysis where the offsite Coulomb interaction induces a screening weakly dependent on filling. These comparisons clearly show that the non-local polarization is the main source of the exotic filling dependence of $U^{\mathrm{DMFT}}$.

It becomes now clear that the similar values of $U^{\mathrm{DMFT}}$ and $U^{\mathrm{cRPA}}$ for $\mathrm{SrVO}_{3}$ is just a consequence of an approximate cancellation of the anti-screening by the non-local polarizations with the screening by the long-range interaction. In addition, $U^{\mathrm{cRPA}} \sim U^{\mathrm{DMFT}} \sim U^{\text {no-wing }}$ for $\mathrm{SrVO}_{3}$ is partly ascribed to the small filling of the $d^{1}$ system where the polarization and screening are not large.

In the previous DMFT studies for the $a b$ initio model, rather large values of $U$ compared to $U^{\text {cRPA }}$ have been needed to reproduce the experimental results (e.g., the insulating behavior of $\mathrm{LaTiO}_{3}[24]$ ). Similarly, for the 2D Hubbard model, the Mott transition takes place at a substantially larger $U$ in the single-site DMFT than in its cluster extension [25]. These aspects are ascribed to the intersite correlation effects ignored in the single-site DMFT with original $U^{\text {cRPA }}$ or $U$. The present scheme with $U^{\mathrm{DMFT}}$ at least partially takes account of the off-site effects and will improve the results of the DMFT. The vertex corrections ignored in the RPA form have been estimated to be small for the conventional cRPA [1]. For the present case, this estimate is left for future studies.

-Conclusion. We have examined a scheme to evaluate the effective onsite interaction $U^{\mathrm{DMFT}}$ for the DMFT. Through the analysis based on the Hubbard model, we have found unexpectedly an anti-screening effect induced by non-local polarizations, which competes with the screening effects caused by the off-site Coulomb interaction in real materials. The anti-screening causes a non-trivial filling dependence of $U^{\mathrm{DMFT}}$ and increases the effective interaction. Combining the present method with DFT, we have indeed shown that $U^{\mathrm{DMFT}}$ for $\mathrm{SrVO}_{3}$ exhibits non-trivial filling dependence if the chemical potential is varied.

-Acknowledgments. We would like to thank Takashi
TABLE I: Onsite bare $(\mathbf{v})$, cRPA $\left(\mathbf{U}^{\text {cRPA }}\right)$, present-scheme $\left(\mathbf{U}^{\mathrm{DMFT}}\right)$, and full-RPA $(\mathbf{W})$ interaction parameters calculated for $\mathrm{SrVO}_{3}$. The unit of energy is eV. The method was implemented in two codes, Tokyo Ab initio Program Package 22] (left values) and the Vienna $A b$ initio Simulation Package 23] (right ones), which yield almost identical values for $\mathbf{U}^{\mathrm{cRPA}}$. Otherwise, the latter values are generally $5-10 \%$ larger than those of the former, since the exact shape of the orbitals is used in VASP.

\begin{tabular}{ccccc}
\hline \hline & $\mathbf{v}$ & $\mathbf{U}^{\mathrm{cRPA}}$ & $\mathbf{U}^{\mathrm{DMFT}}$ & $\mathbf{W}$ \\
\hline$U$ & $15.0,16.0$ & $3.39,3.36$ & $3.33,3.46$ & $0.97,1.12$ \\
$U^{\prime}$ & $13.7,14.8$ & $2.34,2.35$ & $2.27,2.47$ & $0.25,0.30$ \\
$J$ & $0.59,0.55$ & $0.47,0.49$ & $0.47,0.47$ & $0.33,0.39$ \\
\hline \hline
\end{tabular}

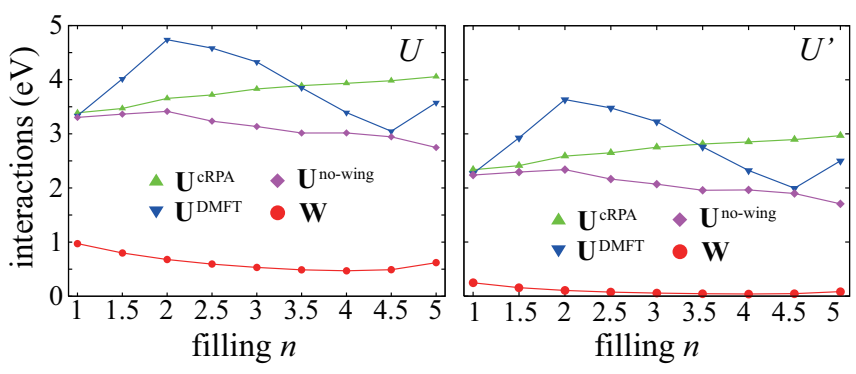

FIG. 2: (color online) Filling dependence of intra-orbital (left) and inter-orbital (right) screened Coulomb repulsion of $\mathrm{SrVO}_{3}$ evaluated within full RPA, cRPA, present scheme $\left(\mathbf{U}^{\mathrm{DMFT}}\right)$, and "no-wing" methods, which are calculated with TAPP [22].

Miyake for fruitful discussions. This work was supported by Grants-in-Aid for Scientific Research (No. 22740215, 22104010, 23110708, 23340095, 22340090) from MEXT and JST-PRESTO, Japan and the Austrian Science fund through F41 (SFB ViCoM) and I597-16.

[1] M. Imada and T. Miyake, J. Phys. Soc. Jpn. 79, 112001 (2010).

[2] G. Kotliar, S. Y. Savrasov, K. Haule, V. S. Oudovenko, O. Parcollet, and C.A.Marianetti, Rev. Mod. Phys. 78 865 (2006).

[3] Y. Imai, I. Solovyev and M. Imada, Phys. Rev. Lett. 95, 176405 (2005).

[4] F. Aryasetiawan, M. Imada, A. Georges, G. Kotliar, S. Biermann, and A. I. Lichtenstein, Phys. Rev. B 70, 195104 (2004).

[5] N. Marzari and D. Vanderbilt, Phys. Rev. B 56, 12847 (1997); I. Souza, N. Marzari, and D. Vanderbilt, ibid. 65, 035109 (2001).

[6] T. Miyake and F. Aryasetiawan, Phys. Rev. B 77, 085122 (2008).

[7] T. Miyake and F. Aryasetiawan, and M. Imada, Phys. Rev. B 80, 155134 (2009). 
[8] K. Nakamura, Y. Yoshimoto, Y. Nohara, and M. Imada, J. Phys. Soc. Jpn. 79, 123708 (2010).

[9] K. Nakamura, T. Koretsune, and R. Arita, Phys. Rev. B 80, 174420 (2009).

[10] K. Nakamura, R. Arita, and M. Imada, J. Phys. Soc. Jpn. 77, 093711 (2008).

[11] T. Miyake, K. Nakamura, R. Arita, and M.Imada, J. Phys. Soc. Jpn. 79, 044705 (2010).

[12] H. Shinaoka, T. Misawa, K. Nakamura, and M. Imada, J. Phys. Soc. Jpn. 81, 034701 (2012).

[13] R. Arita, J. Kuneš, A. V. Kozhevnikov, A. G. Eguiluz, and M. Imada, Phys. Rev. Lett. 108, 086403 (2012).

[14] Y. Nomura, K. Nakamura, and R. Arita, Phys. Rev. B 85, 155452 (2012).

[15] W. Metzner and D. Vollhardt, Phys. Rev. Lett. 62, 324 (1989).

[16] A. Georges and G. Kotliar, Phys. Rev. B 45, 6479 (1992).

[17] A. Kutepov, K. Haule, S. Y. Savrasov, and G. Kotliar, Phys. Rev. B 82, 045105 (2010).

[18] See Supplemental Materials at http://@@@.

[19] The quantity $\tilde{U}+\frac{\tilde{U}}{1+\tilde{U} A}$ is nearly equal to the fully screened Coulomb interaction, which is a smooth function of filling.

[20] K. Held, Adv. Phys. 56, 829 (2007).

[21] We specify the "wing" or two-center components of the polarization matrix in the Wannier basis as

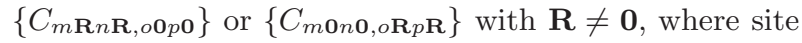
indices are dropped because the unit cell of $\mathrm{SrVO}_{3}$ contains only one $\mathrm{V}$ site. In the analysis on the Hubbard model, this corresponds to $\mathbf{B}$ and $\mathbf{B}^{\mathrm{T}}$ in Eq. (S.8) in Ref. [18].

[22] J. Yamauchi, M. Tsukada, S. Watanabe, and O. Sugino, Phys. Rev. B 54, 5586 (1996).

[23] G. Kresse and J. Furthmüller, Phys. Rev. B 54, 11169 (1996).

[24] E. Pavarini, S. Biermann, A. Poteryaev, A. I. Lichtenstein, A. Georges, and O. K. Andersen, Phys. Rev. Lett. 92, 176403 (2004).

[25] Y. Z. Zhang and M. Imada, Phys. Rev. B 76, 045108 (2007). 


\section{Supplemental Materials}

\section{S.1 DERIVATION OF EQS. (12) AND (14)}

An RPA fully-screened interaction $W$ may be expressed as

$$
\overline{\mathbf{W}}=\overline{\boldsymbol{\epsilon}}^{-1} \overline{\mathbf{U}}, \quad \overline{\boldsymbol{\epsilon}}=\mathbf{1}-\overline{\mathbf{U}} \overline{\boldsymbol{\chi}}_{0} .
$$

Here $\overline{\mathbf{X}}=\left[\overline{\mathbf{W}}, \overline{\mathbf{U}}, \bar{\chi}_{0}, \overline{\boldsymbol{\epsilon}}\right]$ are $2 N \times 2 N$ matrices decomposed into their spin channels according to

$$
\overline{\mathbf{X}}=\left(\begin{array}{l}
\mathbf{X}_{\uparrow \uparrow} \mathbf{X}_{\uparrow \downarrow} \\
\mathbf{X}_{\downarrow \uparrow} \mathbf{X}_{\downarrow \downarrow}
\end{array}\right) \text {. }
$$

With this decomposition, $\overline{\mathbf{U}}$ and $\bar{\chi}_{0}$ are written as

$$
\overline{\mathbf{U}}=\left(\begin{array}{cc}
\mathbf{0} & \mathbf{U} \\
\mathbf{U} & \mathbf{0}
\end{array}\right) \text { and } \bar{\chi}_{0}=\left(\begin{array}{cc}
\frac{1}{2} \boldsymbol{\chi}_{0} & \mathbf{0} \\
\mathbf{0} & \frac{1}{2} \boldsymbol{\chi}_{0}
\end{array}\right) \text {, }
$$

respectively, where $\mathbf{U}$ is a diagonal matrix with elements $U$ and $\chi_{0}$ is a real-space polarization matrix. In the Hubbard model, only the onsite components $W=\left[\mathbf{W}_{\uparrow \downarrow}\right]_{i i}$ are relevant, which are given by

$$
\begin{aligned}
W & =\left[\epsilon_{\uparrow \uparrow}^{-1} \mathbf{U}_{\uparrow \downarrow}+\epsilon_{\uparrow \downarrow}^{-1} \mathbf{U}_{\downarrow \downarrow}\right]_{i i} \\
& =\left[\epsilon_{\uparrow \uparrow}^{-1} \mathbf{U}\right]_{i i} .
\end{aligned}
$$

According to Eqs. (5.9) and (5.10) in Ref. S1], the inverse dielectric matrix in the $\uparrow \uparrow$ spin channel is $(\mathbf{1}-$ $\left.\left(\frac{1}{2} \mathbf{U} \chi_{0}\right)^{2}\right)^{-1}$ so that we obtain

$$
W=\left[\left(\mathbf{1}-\left(\frac{1}{2} \mathbf{U} \boldsymbol{\chi}_{0}\right)^{2}\right)^{-1} \mathbf{U}\right]_{i i} .
$$

This equation is also written as

$$
W=\left[\mathbf{U}+\frac{1}{2} \mathbf{U}\left(\boldsymbol{\chi}_{C}-\boldsymbol{\chi}_{S}\right) \mathbf{U}\right]_{i i}
$$

with $\chi_{C}\left(\chi_{S}\right)$ being the charge (spin) susceptibility given by $\chi_{C}=\left(\mathbf{1}-\frac{1}{2} \boldsymbol{\chi}_{0} \mathbf{U}\right)^{-1} \frac{1}{2} \boldsymbol{\chi}_{0}\left[\boldsymbol{\chi}_{S}=\left(\mathbf{1}+\frac{1}{2} \boldsymbol{\chi}_{0} \mathbf{U}\right)^{-1} \frac{1}{2} \boldsymbol{\chi}_{0}\right]$. In line with $a b$ initio methods, which only take charge fluctuations into account, we consider the term related to $\chi_{C}$ only; the resulting expression for $W$ is

$$
\begin{aligned}
W & =\left[\mathbf{U}+\frac{1}{2} \mathbf{U} \boldsymbol{\chi}_{C} \mathbf{U}\right]_{i i} \\
& =\left[\mathbf{U}+\frac{1}{2} \mathbf{U}\left(\mathbf{1}-\frac{1}{2} \boldsymbol{\chi}_{0} \mathbf{U}\right)^{-1} \frac{1}{2} \boldsymbol{\chi}_{0} \mathbf{U}\right]_{i i} \\
& =\left[\mathbf{U}+\tilde{\mathbf{U}}\left(\mathbf{1}-\boldsymbol{\chi}_{0} \tilde{\mathbf{U}}\right)^{-1} \boldsymbol{\chi}_{0} \tilde{\mathbf{U}}\right]_{i i} \\
& =\left[\tilde{\mathbf{U}}+\left(\mathbf{1}+\tilde{\mathbf{U}} \boldsymbol{\chi}_{0}+\left(\tilde{\mathbf{U}} \boldsymbol{\chi}_{0}\right)^{2}+\cdots\right) \tilde{\mathbf{U}}\right]_{i i} \\
& =\left[\tilde{\mathbf{U}}+\left(\mathbf{1}-\tilde{\mathbf{U}} \boldsymbol{\chi}_{0}\right)^{-1} \tilde{\mathbf{U}}\right]_{i i} \\
& =\tilde{U}+\tilde{W},
\end{aligned}
$$

where $\tilde{U}=U / 2$ and $\tilde{W}=\left[\left(\mathbf{1}-\tilde{\mathbf{U}} \boldsymbol{\chi}_{0}\right)^{-1} \tilde{\mathbf{U}}\right]_{i i}$.

We now decompose the total polarization $\chi_{0}$ into the two parts,

$$
\chi_{0}^{t}=\left(\begin{array}{cc}
-A & \mathbf{0} \\
\mathbf{0} & \mathbf{0}
\end{array}\right) \text { and } \boldsymbol{\chi}_{0}^{\prime}=\left(\begin{array}{cc}
0 & \mathbf{B}^{\mathrm{T}} \\
\mathbf{B} & \chi_{0}^{\prime \prime}
\end{array}\right),
$$

where $\mathbf{B}=\left(B_{1}, B_{2}, \cdots, B_{N-1}\right)^{\mathrm{T}}$ and $\chi_{0}^{\prime \prime}$ is an $(N-$ $1) \times(N-1)$ matrix. Then, replacing $\chi_{0}$ with $\chi_{0}^{\prime}$ in Eqs. (S.17), we obtain

$$
U^{\mathrm{DMFT}}=\tilde{U}+\tilde{U}^{\mathrm{DMFT}}
$$

with

$$
\tilde{U}^{\mathrm{DMFT}}=\left[\left(\mathbf{1}-\tilde{\mathbf{U}} \boldsymbol{\chi}_{0}^{\prime}\right)^{-1} \tilde{\mathbf{U}}\right]_{11} .
$$

The above derivation of $U^{\mathrm{DMFT}}$ is based on the screening approach of Eq. (2). On the other hand, $\tilde{U}^{\mathrm{DMFT}}$ can also be obtained in the unscreening approach of Eq. (3) as

$$
\tilde{U}^{\mathrm{DMFT}}=\left[\tilde{\mathbf{W}}\left(\mathbf{1}+\boldsymbol{\chi}_{0}^{t} \tilde{\mathbf{W}}\right)^{-1}\right]_{11}=\frac{\tilde{W}}{1-A \tilde{W}} .
$$

Eqs. (S.9) and (S.11) give Eq. (12) in the main text. Again using Eqs. (5.9) and (5.10) in Ref. [S1], Eq. (S.10) is further recast into

$$
\tilde{U}^{\mathrm{DMFT}}=\frac{1}{1-\tilde{U}^{2} \mathbf{B}^{\mathrm{T}}\left(\mathbf{1}-\tilde{U} \boldsymbol{\chi}_{0}^{\prime \prime}\right)^{-1} \mathbf{B}} \tilde{U} .
$$

Hence, up to the second order in $\left\{B_{n}\right\}$, we obtain

$$
U^{\mathrm{DMFT}} \sim \tilde{U}+\left(1+\frac{\tilde{U}^{2} \mathbf{B}^{\mathrm{T}} \mathbf{B}}{1+\tilde{U} A}\right) \tilde{U},
$$

which is equivalent to Eq. (14).

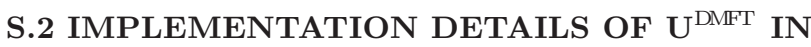 THE PLANE-WAVE BASIS-SET CODE AND COMPUTATIONAL RESULTS}

Here, we describe implementation details for the $a b$ initio $\mathbf{U}^{\mathrm{DMFT}}$ calculations. The calculation is performed with the norm-conserving pseudopotential and plane-wave basis set and the projector augmented wave method, respectively [S2, S3]. In the plane-wave basis-set calculation, two different cutoffs for the plane waves are conventionally used; the low-momentum cutoff glow for the polarization function and the high-momentum cutoff $\mathrm{g}_{\text {cut }}^{\text {high }}$ for orbitals. In general, the structure of the polarization function in real space is smooth compared to that of the wavefunction, so we can employ the smaller cutoff and it considerably reduces the computational cost. In the $\mathbf{U}^{\mathrm{DMFT}}$ calculation in Eq. (11) in the main text, however, we should be careful about the use of the two different cutoffs. 
The Dyson equation Eq. (10) is written in the momentum space with the double Fourier transform [S4] as

$$
\begin{aligned}
& W_{\mathbf{g}_{1} \mathbf{g}_{2}}=U_{\mathbf{g}_{1} \mathbf{g}_{2}}^{\mathrm{DMFT}}+\sum_{\mathbf{g}_{3} \mathbf{g}_{4}} U_{\mathbf{g}_{1} \mathbf{g}_{3}}^{\mathrm{DMMT}} \chi_{\mathbf{g}_{3} \mathbf{g}_{4}} W_{\mathbf{g}_{4} \mathbf{g}_{2}}\left(\left|\mathbf{g}_{i}\right|<\mathrm{g}_{\text {cut }}^{\text {low }}\right) \\
& W_{\mathbf{g}_{1} \mathbf{g}_{2}}=U_{\mathbf{g}_{1} \mathbf{g}_{2}}^{\mathrm{DMFT}}=v_{\mathbf{g}_{1}} \delta_{\mathbf{g}_{1} \mathbf{g}_{2}}\left(\mathrm{~g}_{\text {cut }} \leq\left|\mathbf{g}_{i}\right| \leq \mathrm{g}_{\text {cut }}^{\text {high }}\right),
\end{aligned}
$$

where $\mathbf{g}_{1}-\mathbf{g}_{4}$ are reciprocal wave vectors associated with the superlattice [S5] and $v_{\mathbf{g}}=4 \pi /|\mathbf{g}|^{2}$ is the Fourier transform of the bare Coulomb interaction $v$. In Eq. (S.15) we have used the fact that $\chi_{\mathbf{g g}^{\prime}}^{\text {imp }}$ vanishes outside $g_{\text {cut }}$.

Recognizing this aspect, we define the low- and highmomentum contributions for $W_{\mu \nu}$, defined in Eq. (10), as

$$
\begin{gathered}
W_{\mu \nu}^{\text {dow }}=\frac{1}{V} \sum_{\mathbf{g g}{ }^{\prime}}^{\text {low }}\left\langle\phi_{m 00}\left|e^{i \mathbf{g r}}\right| \phi_{n 00}\right\rangle W_{\mathbf{g g}}\left\langle\left\langle\phi_{000} \mid e^{-i \mathbf{g}^{\prime} \mathbf{r}^{\prime}} \phi_{p 00}\right\rangle(\mathrm{S} .16)\right. \\
W_{\mu \nu}^{\text {high }}=\frac{1}{V} \sum_{\mathbf{g}}^{\text {high }}\left\langle\phi_{m 00}\left|e^{i \mathbf{g r}}\right| \phi_{n 00}\right\rangle v_{\mathbf{g}}\left\langle\phi_{o 00}\left|e^{-i \mathbf{g r}^{\prime}}\right| \phi_{p 00}\right\rangle(\mathrm{S} .17)
\end{gathered}
$$

Here, $V$ is the crystal volume and $W_{\mu \nu}=W_{\mu \nu}^{\text {low }}+W_{\mu \nu}^{\text {high }}$. The sum in Eq. (S.14) is taken for the reciprocal vector within $\mathrm{g}_{\text {cut }}^{\text {low }}$, while the sum in Eq. (S.15) runs over the reciprocal vector for $\mathrm{g}_{\text {cut }}^{\text {low }} \leq|\mathrm{g}| \leq \mathrm{g}_{\text {cut }}^{\text {high }}$. Similarly, $U_{\mu \nu}^{\mathrm{DMFT}}$ is written as the sum of $U_{\mu \nu}^{\mathrm{DMFT}-\text { low }}$ and $U_{\mu \nu}^{\mathrm{DMFT}-h i g h}$. Inserting Eq. (S.14) into Eq. (S.16) with the double Fourier transform of $\chi_{0}^{\text {imp }}$, we obtain

$$
W_{\mu \nu}^{\text {low }}=U_{\mu \nu}^{\text {DMFT-low }}+\sum_{\mu^{\prime} \nu^{\prime}} U_{\mu \mu^{\prime}}^{\text {DMFT-low }} C_{\mu^{\prime} \nu^{\prime}} W_{\nu^{\prime} \nu}^{\text {low }}(\mathrm{S}
$$

or in the matrix form

$$
\mathbf{W}^{\text {low }}=\mathbf{U}^{\text {DMFT-low }}+\mathbf{U}^{\text {DMFT-low }} \mathbf{C W}^{\text {low }} .
$$

Since $\mathbf{U}^{\mathrm{DMFT}}=\mathbf{U}^{\mathrm{DMFT}-\mathrm{low}}+\mathbf{U}^{\mathrm{DMFT}-\text { high }}$, after some manipulations, we obtain

$$
\mathbf{U}^{\mathrm{DMFT}}=\mathbf{W}^{\text {low }}\left(\mathbf{1}+\mathbf{C W}^{\text {low }}\right)^{-1}+\mathbf{V}^{\text {high }}
$$

with $\mathbf{V}^{\text {high }}\left(=\mathbf{W}^{\text {high }}=\mathbf{U}^{\text {DMFT-high }}\right)$ being the matrix of $v$ at high momenta Eq. (S.17). In the actual calculation, this expression is used.

As a note on the numerical calculation, we remark some details for calculating the polarization function in a metallic system. The target-band polarization $\chi_{0}^{t b}\left(\mathbf{r}, \mathbf{r}^{\prime}\right)$ in Eq. (4) in the main text is given in the momentum space with the double Fourier transform as

$$
\begin{aligned}
\chi_{\mathbf{G G}^{\prime}}^{t b}(\mathbf{q})=2 \sum_{\mathbf{k}} \sum_{\alpha \beta}^{\epsilon t b} \frac{f_{\beta \mathbf{k}+\mathbf{q}}-f_{\alpha \mathbf{k}}}{\epsilon_{\beta \mathbf{k}+\mathbf{q}}-\epsilon_{\alpha \mathbf{k}}}\left\langle\psi_{\alpha \mathbf{k}}\left|e^{-i(\mathbf{q}+\mathbf{G}) \cdot \mathbf{r}}\right| \psi_{\beta \mathbf{k}+\mathbf{q}}\right\rangle \\
\quad \times\left\langle\psi_{\beta \mathbf{k}+\mathbf{q}}\left|e^{i\left(\mathbf{q}+\mathbf{G}^{\prime}\right) \cdot \mathbf{r}^{\prime}}\right| \psi_{\alpha \mathbf{k}}\right\rangle . \quad(\mathrm{S} .21)
\end{aligned}
$$

Here, $\mathbf{G}$ is a reciprocal lattice vector for the primitive lattice and $\mathbf{q}$ is a wave vector in the first Brillouin zone. $\left\{\psi_{\alpha \mathbf{k}}\right\},\left\{\epsilon_{\alpha \mathbf{k}}\right\}$, and $\left\{f_{\alpha \mathbf{k}}\right\}$ are the Bloch states, their energies, and occupancies, respectively, and the band summation runs over the target bands only. In the calculation of $\chi_{\mathbf{G G}^{\prime}}^{t b}(\mathbf{q})$ of the metallic system, the $\mathbf{k}$ integral on the right hand must be performed carefully, because the expression includes a numerical instability due to the Lindhard part. To avoid the instability, we use the Wannier interpolation scheme [S6]; we interpolate the original kpoint data (of about $10 \times 10 \times 10$ ) for the eigenvalues $\left\{\epsilon_{\alpha \mathbf{k}}\right\}$ and interstate matrix elements $\left\{\left\langle\psi_{\beta \mathbf{k}+\mathbf{q}}\left|e^{i(\mathbf{q}+\mathbf{G}) \mathbf{r}}\right| \psi_{\alpha \mathbf{k}}\right\rangle\right\}$, to obtain the data on a denser $\mathbf{k}$ grid (about $30 \times 30 \times 30$ ). After such an interpolation, the $\mathbf{k}$ integration is performed with the generalized tetrahedron method [S7] to obtain both, real and imaginary parts of $\chi_{\mathbf{G G}^{\prime}}^{t b}(\mathbf{q})$.

We also need a careful treatment of poles at $\epsilon_{\beta \mathbf{k}+\mathbf{q}}=$ $\epsilon_{\alpha \mathbf{k}}$ in Eq. (S.21), for which we rewrite

$$
\frac{f_{\beta \mathbf{k}+\mathbf{q}}-f_{\alpha \mathbf{k}}}{\epsilon_{\beta \mathbf{k}+\mathbf{q}}-\epsilon_{\alpha \mathbf{k}}} \sim \delta\left(\frac{\epsilon_{\beta \mathbf{k}+\mathbf{q}}+\epsilon_{\alpha \mathbf{k}}}{2}-\epsilon_{\mathrm{F}}\right),
$$

Based on the central-difference approximation of the Fermi-Dirac function with The Fermi level $\epsilon_{\mathrm{F}}$. Switching to the $\delta$ function in Eq. (S.22) is performed in the threshold $\left|\epsilon_{\beta \mathbf{k}+\mathbf{q}}-\epsilon_{\alpha \mathbf{k}}\right|<0.06 \mathrm{eV}$ and the $\delta$ function is treated with a smearing factor of $0.03 \mathrm{eV}$. With the resulting target-band polarization $\chi^{t b}$ and the rest polarization $\chi^{r}$ [S8], the fully screened RPA Coulomb interaction $\mathbf{W}^{\text {low }}$ in Eq. (S.20) is calculated, where the $W_{\mathbf{G G}^{\prime}}(\mathbf{q})$ interaction at $\mathbf{q} \rightarrow \mathbf{0}$ limit is treated following Ref. [S1]. The same treatment is applied to the evaluation of the Wannier matrix elements of $C_{\text {mmop }}$ in Eq. (8) [S9]. With all these treatments, the present $\mathbf{U}^{\mathrm{DMFT}}$ calculation ensures the accuracy within several percent.

If not otherwise noted, the density-functional theory calculations for $\mathrm{SrVO}_{3}$ were performed with Tokyo Ab initio Program Package [S10], which is based on the pseudopotential plus plane-wave framework. The exchange-correlation functional is calculated within the generalized-gradient approximation with Perdew-Burke-Ernzerhof (PBE) parameterization [S11], and the Troullier-Martins norm-conserving pseudopotentials [S12] in the Kleinman-Bylander representation S13] is adopted. In the present calculation for Fig. 2 in the main text, the cutoff energies for wavefunctions and polarization functions are set to $49 \mathrm{Ry}$ and $25 \mathrm{Ry}$, respectively, and we employ $11 \times 11 \times 11 \mathrm{k}$ points. The Brillouinzone integrals are evaluated using the generalized tetrahedron method [S7] after interpolation to a $33 \times 33 \times 33 \mathrm{k}$ mesh.

Where noted, additional calculations were performed using the Vienna Ab initio Simulation Package (VASP), using projector augmented waves and the local density approximation. The plane wave cutoff energies for the orbitals and response functions were set to $414 \mathrm{eV}$ (30 Ry) and $250 \mathrm{eV}$ (18 Ry), respectively. Extrapolation to a high energy cutoff $(500 \mathrm{eV})$ was performed using Eq. (S.20). 
In VASP no intermediate extrapolation to a denser kpoint grid was performed. Instead, in Eq. (S.21), the Fermi occupancy function $f(\epsilon)$ was replaced by a Methfessel Paxton smearing function with $\sigma=0.1$ S14], and consistent with metallic screening $\mathbf{W}_{\mathbf{0 0}}(\mathbf{q} \rightarrow 0)$ was set to 0 .

Figure S 1 shows our calculated band structure of $\mathrm{SrVO}_{3}$ (a) and the density of states for the $t_{2 g}$ bands (b). The arrows in the panel (b) indicate the Fermi levels for the fillings $n=1.0$ to 5.0 with the interval 0.5 . We see that the van Hove singularity nearly corresponds to the Fermi level at the filling $n=4.0$.
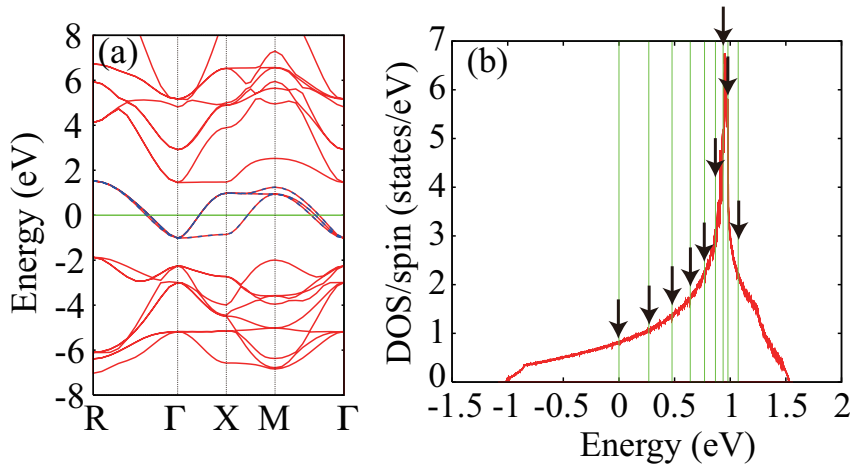

Fig. S 1: (color online) (a) Calculated electronic band structure of $\mathrm{SrVO}_{3}$. The interpolated band dispersions for the $t_{2 g}$ bands are depicted as blue dashed lines, which cross the Fermi level. (b) Calculated density of states for the $t_{2 g}$ bands. Black arrows indicate the Fermi level for the filling $n=1.0-5.0$ from left to right for the values shown in Table S IV

We show in Table $\mathrm{S}$ I and $\mathrm{S} \Pi$ the convergence behavior of $\mathbf{U}^{\mathrm{DMFT}}$ calculated for $\mathrm{SrVO}_{3}$ against the sampling $\mathbf{k}$ points using the Tokyo Ab initio Program Package. The table lists the values for the onsite intra- and inter-orbital Coulomb repulsions $\left(U\right.$ and $\left.U^{\prime}\right)$ and Hund's rule coupling $(J)$. The usual constrained random-phase-approximation

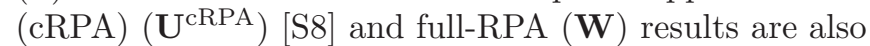
shown for comparison. We see that the results are almost converged at $6 \times 6 \times 6$ or $7 \times 7 \times 7 \mathrm{k}$-point samplings. Despite a less sophisticated interpolation procedure the results using the Vienna Ab initio Simulation Package (VASP) show a very similar convergence behavior. Again the error is reduced to few percent at $7 \times 7 \times 7 \mathrm{k}$-points, although a sizeable scattering prevails in both codes.
Table S III shows the convergence behavior against the cutoff momentum g gut for the polarization function. We see that the convergence is attained around g gow $\sim 25$ Ry. Finally, Table S IV lists the interaction parameters calculated at the fillings $n=1.0-5.0$, which are used for the plot in Fig. 2 in the main text. In this table, we add the "no-wing" data ( $\left.\mathbf{U}^{\text {no-wing }}\right)$. For the definition of $\mathbf{U}^{\text {no-wing, }}$, see the main text.

[S1] R. M. Pick, M. H. Cohen, and R. M. Martin, Phys. Rev. B 1, 910 (1970).

[S2] P.E. Blöchl, Phys. Rev. B 50, 17953 (1994).

[S3] G. Kresse and D. Joubert, Phys. Rev. B 59, 1758 (1999).

[S4] M. S. Hybertsen and S. G. Louie, Phys. Rev. B 35, 5585 (1987).

[S5] When we consider the impurity problem in the real space, the translational symmetry of the primitive lattice is broken. So, we have to express all the quantities in terms of the periodicity of the superlattice. Note that the system has still a translational symmetry of the superlattice, due to the periodic boundary condition. The present derivation follows the superlattice formulation. On the other hand, the fully screened RPA Coulomb interaction W needed in the $\mathbf{U}^{\mathrm{DMFT}}$ calculation can be calculated in the original periodicity of the primitive lattice, because $\mathbf{W}$ itself is free from the impurity problem. In the present work, we calculate $\mathbf{W}$ for the original primitive lattice.

[S6] X. Wang, J. R. Yates, I. Souza, and D. Vanderbilt, Phys. Rev. B 74, 195118 (2006); J. R. Yates, X. Wang, D. Vanderbilt, and I. Souza, Phys. Rev. B 75, 195121 (2007).

[S7] T. Fujiwara, S. Yamamoto, and Y. Ishii, J. Phys. Soc. Jpn. 72, 777 (2003); Y. Nohara, S. Yamamoto, and T. Fujiwara, Phys. Rev. B 79, 195110 (2009); J. Rath and A. J. Freeman, Phys. Rev. B 11, 2109 (1975).

[S8] K. Nakamura, R. Arita, and M. Imada, J. Phys. Soc. Jpn. 77, 093711 (2008).

[S9] We first evaluated the $\mathbf{k}$ integral by the generalized tetrahedron method [S7] in a finer mesh with the interpolation technique, then we performed the sum over $\mathbf{q}$ in the original mesh.

[S10] J. Yamauchi, M. Tsukada, S. Watanabe, and O. Sugino, Phys. Rev. B 54, 5586 (1996).

[S11] J. P. Perdew, K. Burke, and M. Ernzerhof, Phys. Rev. Lett. 77, 3865 (1996).

[S12] N. Troullier and J. L. Martins, Phys. Rev. B 43, 1993 (1991).

[S13] L. Kleinman and D. M. Bylander, Phys. Rev. Lett. 48, 1425 (1982).

[S14] M. Methfessel and A. T. Paxton, Phys. Rev. B 40, 3616 (1989). 
Table S I: Convergence behavior of $\mathbf{U}^{\text {cRPA }}, \mathbf{U}^{\mathrm{DMFT}}$, and $\mathbf{W}$ to the sampling $k$ points of $\mathrm{SrVO}_{3}$ for the Tokyo Ab initio Program Package. The cutoff energy for polarization function is $25 \mathrm{Ry}$.

\begin{tabular}{rccccccccc}
\hline \hline & \multicolumn{3}{c}{$\mathbf{U}^{\mathrm{cRPA}}$} & \multicolumn{3}{c}{$\mathbf{U}^{\mathrm{DMFT}}$} & \multicolumn{3}{c}{$\mathbf{W}$} \\
\hline & $U$ & $U^{\prime}$ & $J$ & $U$ & $U^{\prime}$ & $J$ & $U$ & $U^{\prime}$ & $J$ \\
\hline $5 \times 5 \times 5$ & 3.40 & 2.34 & 0.47 & 3.48 & 2.41 & 0.41 & 0.93 & 0.23 & 0.33 \\
$6 \times 6 \times 6$ & 3.50 & 2.45 & 0.47 & 3.44 & 2.37 & 0.47 & 0.98 & 0.25 & 0.33 \\
$7 \times 7 \times 7$ & 3.42 & 2.37 & 0.47 & 3.37 & 2.30 & 0.47 & 0.97 & 0.25 & 0.33 \\
$8 \times 8 \times 8$ & 3.32 & 2.27 & 0.47 & 3.26 & 2.20 & 0.48 & 0.96 & 0.24 & 0.33 \\
$9 \times 9 \times 9$ & 3.27 & 2.22 & 0.47 & 3.22 & 2.16 & 0.48 & 0.97 & 0.25 & 0.33 \\
$10 \times 10 \times 10$ & 3.44 & 2.38 & 0.47 & 3.39 & 2.33 & 0.47 & 0.98 & 0.25 & 0.33 \\
$11 \times 11 \times 11$ & 3.39 & 2.34 & 0.47 & 3.33 & 2.27 & 0.47 & 0.97 & 0.25 & 0.33 \\
\hline \hline
\end{tabular}

Table S II: Convergence behavior of $\mathbf{U}^{\mathrm{cRPA}}, \mathbf{U}^{\mathrm{DMFT}}$, and $\mathbf{W}$ to the sampling $k$ points of $\mathrm{SrVO}_{3}$ for the Vienna Ab initio Simulation Package.

\begin{tabular}{rcccccccccc}
\hline \hline \multicolumn{4}{c}{$\mathbf{U}^{\mathrm{cRPA}}$} & \multicolumn{3}{c}{$\mathbf{U}^{\mathrm{DMFT}}$} & \multicolumn{3}{c}{$\mathbf{W}$} \\
\hline & $U$ & $U^{\prime}$ & $J$ & $U$ & $U^{\prime}$ & $J$ & $U$ & $U^{\prime}$ & $J$ \\
\hline $3 \times 3 \times 3$ & 3.45 & 2.43 & 0.50 & 6.38 & 5.38 & 0.48 & 1.02 & 0.23 & 0.38 \\
$4 \times 4 \times 4$ & 3.31 & 2.30 & 0.49 & 5.25 & 4.26 & 0.47 & 1.00 & 0.22 & 0.38 \\
$5 \times 5 \times 5$ & 3.31 & 2.30 & 0.49 & 3.94 & 2.95 & 0.47 & 1.07 & 0.26 & 0.39 \\
$6 \times 6 \times 6$ & 3.35 & 2.34 & 0.49 & 3.50 & 2.51 & 0.47 & 1.11 & 0.29 & 0.39 \\
$7 \times 7 \times 7$ & 3.38 & 2.36 & 0.49 & 3.51 & 2.53 & 0.47 & 1.17 & 0.34 & 0.40 \\
$8 \times 8 \times 8$ & 3.36 & 2.35 & 0.49 & 3.46 & 2.47 & 0.47 & 1.12 & 0.30 & 0.39 \\
$9 \times 9 \times 9$ & - & - & - & 3.42 & 2.43 & 0.47 & 1.10 & 0.29 & 0.39 \\
$10 \times 10 \times 10$ & - & - & - & 3.42 & 2.43 & 0.47 & 1.11 & 0.30 & 0.39 \\
$11 \times 11 \times 11$ & - & - & - & 3.48 & 2.49 & 0.47 & 1.14 & 0.31 & 0.39 \\
\hline \hline
\end{tabular}

Table S III: Convergence behavior of $\mathbf{U}^{\mathrm{cRPA}}, \mathbf{U}^{\mathrm{DMFT}}$, and $\mathbf{W}$ to the cutoff energy for polarization function $\mathrm{g}_{\text {cut }}^{\text {low }}$ for the Tokyo Ab initio Program Package. The sampling $k$ points are fixed at $7 \times 7 \times 7$ and, in the interpolation of the polarization calculation, the $21 \times 21 \times 21 k$-grid is employed.

\begin{tabular}{lccccccccc}
\hline \hline & \multicolumn{3}{c}{$\mathbf{U}^{\mathrm{cRPA}}$} & \multicolumn{3}{c}{$\mathrm{U}^{\mathrm{DMFT}}$} & \multicolumn{3}{c}{$\mathbf{W}$} \\
\hline & $U$ & $U^{\prime}$ & $J$ & $U$ & $U^{\prime}$ & $J$ & $U$ & $U^{\prime}$ & $J$ \\
\hline $10 \mathrm{Ry}$ & 3.48 & 2.37 & 0.51 & 3.38 & 2.28 & 0.51 & 1.22 & 0.26 & 0.45 \\
$15 \mathrm{Ry}$ & 3.48 & 2.39 & 0.49 & 3.39 & 2.30 & 0.49 & 1.13 & 0.27 & 0.39 \\
$20 \mathrm{Ry}$ & 3.44 & 2.38 & 0.48 & 3.37 & 2.30 & 0.48 & 1.04 & 0.26 & 0.36 \\
$25 \mathrm{Ry}$ & 3.42 & 2.37 & 0.47 & 3.37 & 2.30 & 0.47 & 0.97 & 0.25 & 0.33 \\
$30 \mathrm{Ry}$ & 3.41 & 2.36 & 0.47 & 3.37 & 2.30 & 0.47 & 0.94 & 0.24 & 0.32 \\
$35 \mathrm{Ry}$ & 3.40 & 2.36 & 0.47 & 3.37 & 2.30 & 0.47 & 0.91 & 0.24 & 0.31 \\
\hline \hline
\end{tabular}


Table S IV: Our calculated $\mathbf{U}^{\mathrm{cRPA}}$, $\mathbf{U}^{\mathrm{DMFT}}$, and $\mathbf{W}$ at fillings $n=1.0-5.0$ (Tokyo Ab initio Program Package). These data are used in Fig. 2 in the main text. The $\mathbf{U}^{\text {no-wing }}$ data are also listed. For the definition of $\mathbf{U}^{\text {no-wing }}$, see the main text.

\begin{tabular}{ccccccccccccc}
\hline \hline & \multicolumn{3}{c}{$\mathbf{U}^{\text {cRPA }}$} & \multicolumn{3}{c}{$\mathbf{U}^{\text {DMFT }}$} & \multicolumn{3}{c}{$\mathbf{U}^{\text {no-wing }}$} & \multicolumn{3}{c}{$\mathbf{W}$} \\
\hline & $U$ & $U^{\prime}$ & $J$ & $U$ & $U^{\prime}$ & $J$ & $U$ & $U^{\prime}$ & $J$ & $U$ & $U^{\prime}$ & $J$ \\
\hline$n=1.0$ & 3.39 & 2.34 & 0.47 & 3.33 & 2.27 & 0.47 & 3.30 & 2.24 & 0.47 & 0.97 & 0.25 & 0.33 \\
$n=1.5$ & 3.47 & 2.41 & 0.47 & 4.01 & 2.93 & 0.48 & 3.36 & 2.29 & 0.48 & 0.80 & 0.16 & 0.29 \\
$n=2.0$ & 3.65 & 2.59 & 0.46 & 4.74 & 3.63 & 0.47 & 3.41 & 2.34 & 0.47 & 0.68 & 0.11 & 0.26 \\
$n=2.5$ & 3.72 & 2.65 & 0.46 & 4.58 & 3.48 & 0.47 & 3.23 & 2.16 & 0.47 & 0.59 & 0.07 & 0.24 \\
$n=3.0$ & 3.83 & 2.75 & 0.45 & 4.33 & 3.23 & 0.46 & 3.14 & 2.07 & 0.46 & 0.53 & 0.06 & 0.22 \\
$n=3.5$ & 3.89 & 2.81 & 0.45 & 3.85 & 2.76 & 0.45 & 3.01 & 1.96 & 0.45 & 0.49 & 0.04 & 0.20 \\
$n=4.0$ & 3.93 & 2.85 & 0.44 & 3.39 & 2.32 & 0.44 & 3.02 & 1.96 & 0.44 & 0.47 & 0.04 & 0.20 \\
$n=4.5$ & 3.98 & 2.90 & 0.44 & 3.05 & 2.00 & 0.43 & 2.94 & 1.90 & 0.43 & 0.50 & 0.05 & 0.20 \\
$n=5.0$ & 4.06 & 2.97 & 0.43 & 3.58 & 2.50 & 0.43 & 2.75 & 1.71 & 0.42 & 0.62 & 0.08 & 0.24 \\
\hline \hline
\end{tabular}

